\title{
Adjuvant treatment with Angong Niuhuang pills in treating traumatic brain damage: a meta-analysis of randomized controlled trials
}

\author{
Peng-Cheng Zhao, Zhen-Shan Huang, Shao-Nian Xu, Yong-Ming Zhang \\ Clinical College of the People's Liberation Army, Anhui Medical University, Hefei, China \\ Contributions: (I) Conception and design: PC Zhao, YM Zhang; (II) Administrative support: YM Zhang; (III) Provision of study materials or patients: \\ PC Zhao, ZS Huang, SN Xu; (IV) Collection and assembly of data: PC Zhao, ZS Huang, SN Xu; (V) Data analysis and interpretation: All authors; (VI) \\ Manuscript writing: All authors; (VII) Final approval of manuscript: All authors. \\ Correspondence to: Yong-Ming Zhang. Clinical College of the People's Liberation Army, Anhui Medical University, No. 424 Changiiang West Road, \\ Shushan District, Hefei, China. Email: zymhf2966@163.com.
}

\begin{abstract}
Backgrounds The Angong Niuhuang pill (ANP) has been widely used in the adjuvant treatment of patients with traumatic brain injury (TBI). However, the efficacy and adverse reactions of this drug are controversial. In this study, it was aimed to evaluate the effectiveness and safety of ANP on patients with TBI by a systematic review and meta-analysis of randomized controlled trials (RCTs).

Methods: PubMed, Embase, Cochrane Library, Chinese Biomedicine Database (CBM), China National Knowledge Infrastructure (CNKI), Chinese Scientific Journals Database (VIP), and Wangfang databases were systematically searched from their establishment until June 2020. RCTs of ANP treating TBI were enrolled. Odds risk (OR) was used to assess the total effective rate and safety and mean difference (MD) and $95 \%$ confidence interval (CI) were used to assess the quantitative data. Tthe included literature's quality was evaluated by RevMan 5.3. The sensitivity and publication bias was evaluated by Stata 16.0.

Results: Twelve studies were identified in this systematic review, including 1,568 participants. The metaanalysis results suggested that ANP combined with routine treatment obviously improved the postoperative GCS $[M D=1.97,95 \%$ CI $(1.22,2.72), \mathrm{P}<0.01]$ and GOS $[\mathrm{OR}=2.28,95 \%$ CI $(1.60,3.22), \mathrm{P}<0.01]$ of patients with TBI. ANP also increased $\mathrm{Mg}^{2+}$ concentration and decreased pulmonary infection. In addition, ANP significantly reduced NSE, gastrointestinal bleeding, and liver and kidney function damage.

Conclusions: Based on limited evidence, ANP adjuvant therapy may have a clinical benefit in improving the prognosis of patients with TBI and reducing the associated complications. At the same time, more studies with larger sample sizes and high quality are required to determine the safety and effectiveness of ANP adjuvant therapy.
\end{abstract}

Keywords: Angong Niuhuang pill (ANP); traumatic brain injury (TBI); meta-analysis; randomized control trial

Submitted Jun 28, 2020. Accepted for publication Sep 29, 2020.

doi: 10.21037/apm-20-1331

View this article at: http://dx.doi.org/10.21037/apm-20-1331

\section{Introduction}

Traumatic brain injury (TBI) poses a serious challenge to public health worldwide, and it is also a main cause of longterm disability and the dead among young and children's patients in developed countries. TBI is a serious threat to human life and quality of life (1). According to the statistics, at least 10 million hospitalizations and deaths are caused by TBI every year worldwide. Although the development of neurosurgical treatment technology has dramatically improved TBI's clinical treatment, the mortality and disability rates remain high $(2,3)$. In addition, surgical 
treatment has an ideal effect, with a variety of complications after the operation, which have a serious impact on the postoperative recovery and prognosis of patients $(4,5)$. Therefore, patients need to choose a scientific and effective treatment after their operation to avoid a secondary TBI, promote the normal function of the whole-body organs, and reduce complications.

Herbal medicine has been widely used in patients with TBI (6). The Angong Niuhuang pill (ANP) was recorded in the Qing Dynasty's medical book Differentiation of Febrile Diseases (7). It has the function of clearing away heat and detoxification, relieving shock, and enlightening, and mainly treats evil heat invagination, pericardiuminduced high fever, restlessness, delirium, turbid phlegm, and infantile wind. It is a well-known first aid medicine in the field of traditional Chinese medicine (8-10). Modern medical research shows that ANP has obvious anticonvulsant, sedative, and brain-protective effects, with a significant effect on TBI $(11,12)$. In the past, there was no meta-analysis research comprehensively assessing the ANP's safety and efficacy in TBI patients. Thus, the relevant studies were collected comprehensively, and based on strict design, a systematic review and meta-analysis was performed to explore ANP adjuvant therapy's impact on the effectiveness and safety of TBI patients, so that a reference for the ANP's clinical application in the treatment of TBI can be provided in the future. We present the following article in accordance with the PRISMA reporting checklist (available at http://dx.doi.org/10.21037/apm-20-1331).

\section{Methods}

\section{Search strategy}

According to the Preferred Reporting Items for Systematic Reviews and Meta-Analyses (PRISMA) guidelines, we performed this meta-analysis (13). PubMed, Embase, Cochrane Library, Chinese Biomedicine Database (CBM), China National Knowledge Infrastructure (CNKI), Chinese Scientific Journals Database (VIP), and Wangfang databases were systematically searched for RCTs published from their establishment until June 2020. This process was used to identify all articles based on the MeSH terms and keywords, namely "Angong Niuhuang pill" and "traumatic brain injury," without considering any limitations during the literature search. To further determine the relevant studies, we also consulted the references of published studies.

\section{Inclusion and exclusion criteria}

Inclusion criteria were as follows: (I) study population: patients with a diagnosis of traumatic craniocerebral injury; (II) intervention: all the included studies were given routine symptomatic support treatment in the control group, and studies in the experimental group were treated with ANP on this basis; (III) comparator: compared the prognosis of patients with ANP and without ANP; (IV) outcome measures: Glasgow Coma Score (GCS) after treatment, Glasgow Outcome Score (GOS), pulmonary infection, gastrointestinal bleeding, hypohepatia, renal insufficiency, $\mathrm{Mg}^{2+}$ concentration, neuron-specific enolase (NSE) concentration; (V) study design: RCTs. Exclusion criteria were as follows: (I) non-clinical RCT and fundamental research; (II) clinical studies without any control groups or subjects who did not meet the inclusion criteria; (III) studies with insufficient data or irrelevant topics.

\section{Data extraction and quality assessment}

Two researchers scrutinized the literature, extracted the data, and evaluated the studies' methodological quality, independently. In cases of a dispute, a third researcher participated in the discussions. The data collected included the first authors' names, publication years, patients (sample size, age, and gender), ANP interventions and controls, ANP regimens, and outcome measures. We assessed the methodological quality of the included trials by the Cochrane risk of bias tool (13).

The following seven items were included in this kind of bias tool: random sequence generation, allocation hiding, blindness of participants and personnel, blindness of result data, incomplete result data, selective reporting, and other biases (14).

\section{Statistical analysis}

Based on the RevMan 5.3 software from the Cochrane collaboration network, the statistical data was expressed with odds risk (OR), $95 \%$ confidence interval (CI), and $\mathrm{I}^{2}$. In cases when studies were less than three, we provided a qualitative description. Heterogeneity was evaluated by $\mathrm{I}^{2}$. When $\mathrm{I}^{2} \leq 50 \%$, the effect value was estimated by the fixedeffects model, and when $\mathrm{I}^{2}>50 \%$, we applied the randomeffects model. Publication bias was evaluated by Begg's rank correlation and Egger's regression tests of Stata 16.0, and 


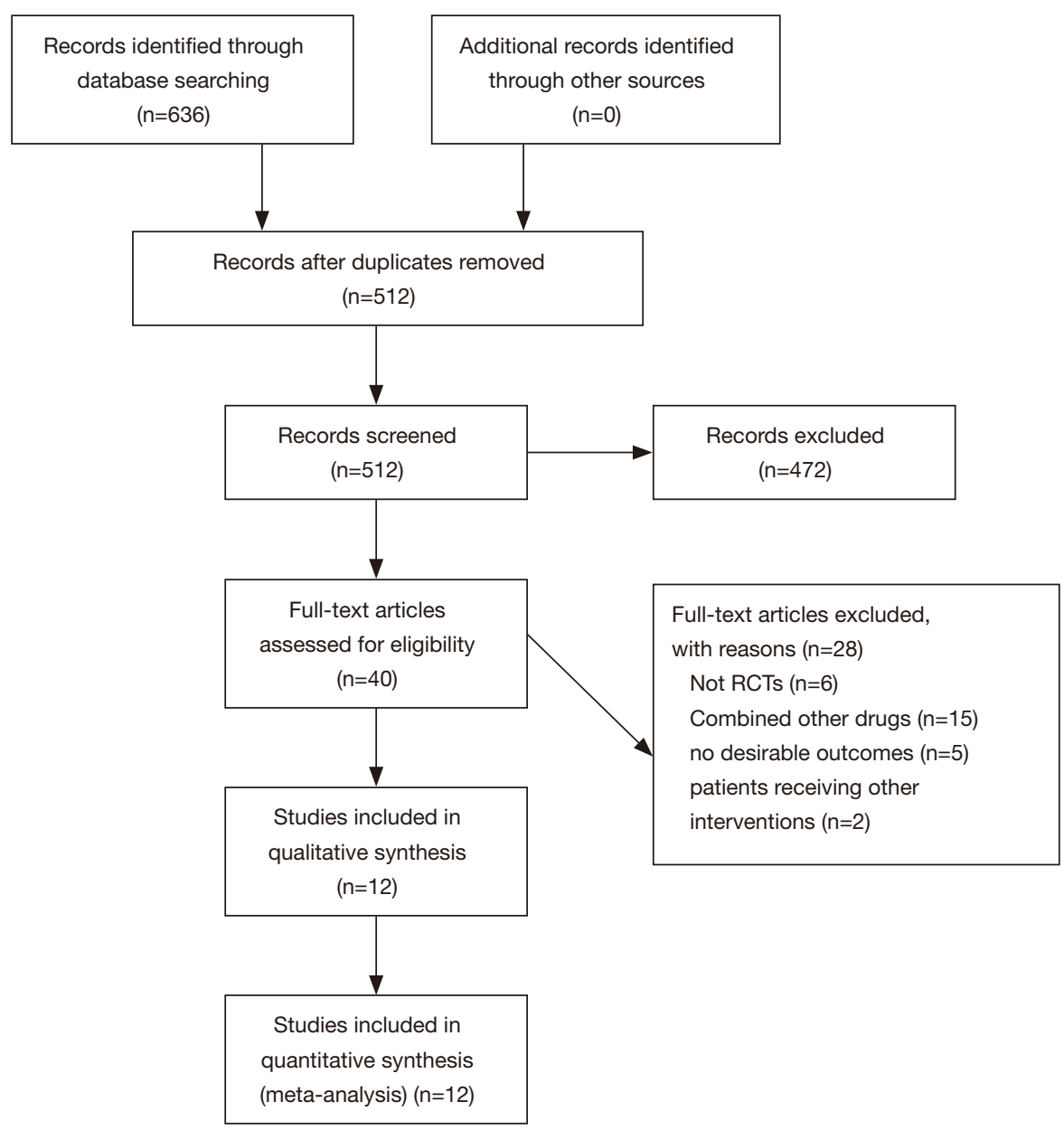

Figure 1 Flow chart of study selection.

when $\mathrm{P}>0.05$, there was no publication bias.

\section{Results}

\section{Basic information of the included articles}

A total of 636 original articles were identified, and 596 articles were ignored after reviewing their titles and brief abstracts. Eventually, 40 full-text papers were evaluated as eligibility. Twenty-eight researches were excluded because of their non-RCT design $(n=6)$, comparison of ANP with other drugs $(n=15)$, lack of desirable outcomes $(n=5)$, and patients receiving other interventions $(\mathrm{n}=2)$. Finally, 12 RCTs $(7,15-25)$ eligible for inclusion criteria were selected (Figure 1).

In total, there were 1,568 patients in 12 studies. These studies had been published between 2009 and 2018, and each trial included 56 to 312 patients. The ANP components used by all patients were the same, and the basic information has no significant differences between two groups. The specific features of the selected trials were listed in Table 1. The evaluation of the outcomes was presented at the end of the treatment in the trials.

\section{Literature quality evaluation}

Figure 2 summarized the bias assessment risk of 12 RCTs. Randomization was mentioned in all studies. However, only eight studies $(7,15,17-19,21,22,25)$ expounded the generation of random sequences. None of the studies described allocation concealment or the blinding of patients or outcome assessments, which may be used to improve the therapeutic effectiveness. Only one study lacked a followup (18). Overall, the selected trials' quality was suboptimal, with an unclear risk of bias (Figure 2). 
Table 1 Baseline characteristic of studies included in this study

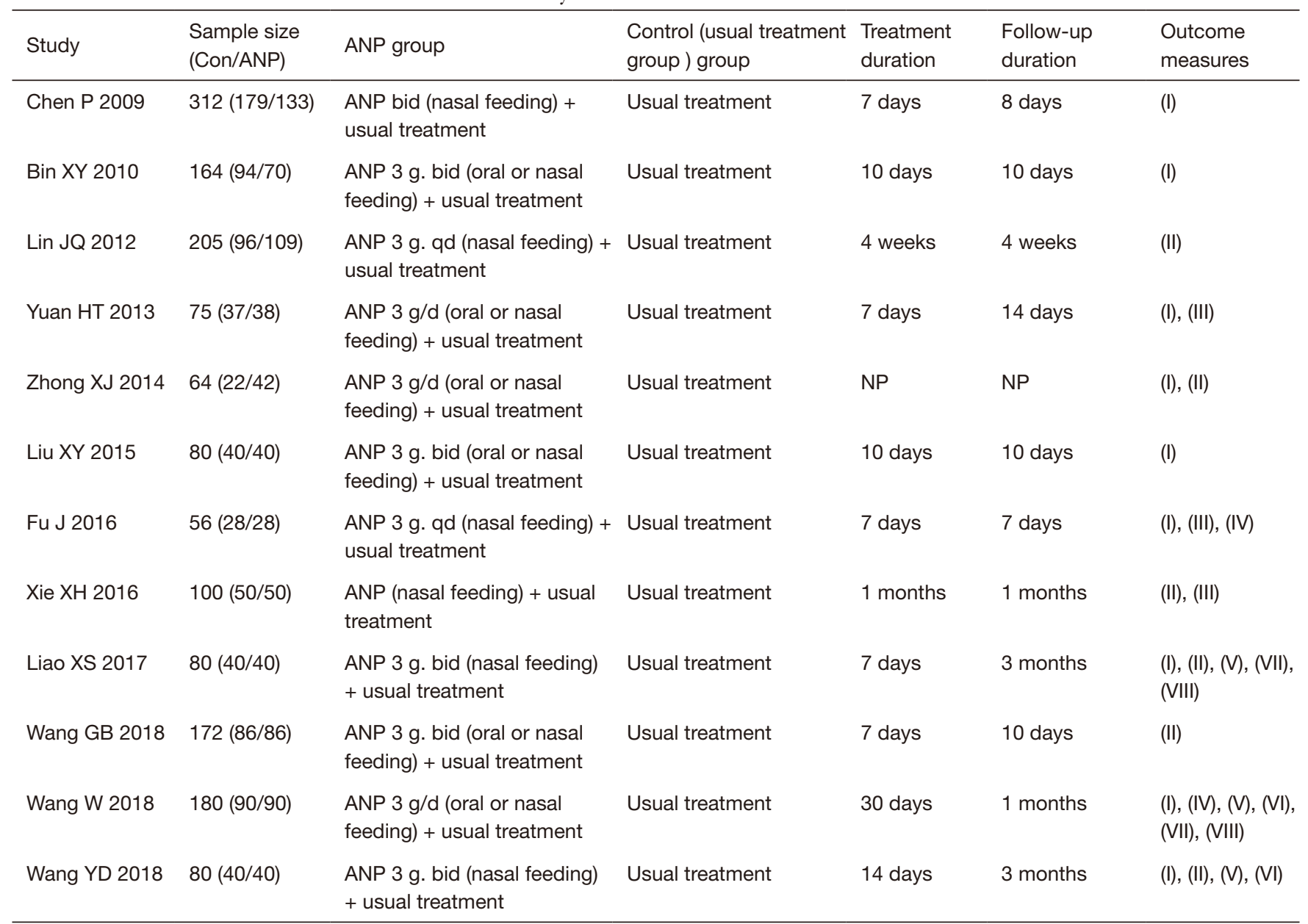

Note: (I) GCS, Glasgow Coma Scale; (II) GOS, Glasgow Outcome Scale; (III) Mg ${ }^{2+}$ concentration; (IV) NSE, neuron-specific enolase concentration; (V) Pulmonary infection; (VI) Gastrointestinal bleeding; (VII) Hypohepatia; (VIII) Renal insufficiency. ANP, Angong Niuhuang pill; NP, not provided.

\section{Meta-analysis of GCS, GOS, $\mathrm{Mg}^{2+}$ concentration, and pulmonary infection}

Nine trials (15-18,20-22,24,25) reported postoperative GCS, with statistical heterogeneity among the studies $\left(\mathrm{P}<0.001, \mathrm{I}^{2}=97 \%\right)$, which was analyzed using the random effect model. The meta-analysis results showed that the GCS in the control group was significantly lower than that in the test group after treating, which has statistically significant difference $[\mathrm{MD}=1.97,95 \% \mathrm{CI}(1.22,2.72)$, $\mathrm{P}<0.01$ ] (Figure 3).

The postoperative GOS was evaluated in six studies $(7,18,19,22,23,25)$, without statistical heterogeneity $(\mathrm{P}=0.53$, $\left.\mathrm{I}^{2}=0 \%\right)$. The results were analyzed by fixed effect model. Compared with the conventional therapy, treatment with
ANP significantly improved the GOS $>3$ rate $[\mathrm{OR}=2.28$, $95 \%$ CI $(1.60,3.23), \mathrm{P}<0.01]$ (Figure 4).

Three studies $(17,23,24)$ reported a concentration of $\mathrm{Mg}^{2+}$ in peripheral blood after treatment, with no statistical heterogeneity among the studies $\left(\mathrm{P}=0.77, \mathrm{I}^{2}=0 \%\right)$. The results were analyzed by fixed effect model. After treatment, the $\mathrm{Mg}^{2+}$ concentration in peripheral blood in the control group was significantly lower than that in the test group, which has statistically significant difference. $[\mathrm{MD}=-0.11$, 95\% CI (-0.16, -0.06), $\mathrm{P}<0.01$ ] (Figure 5).

Three studies $(18,21,22)$ reported pulmonary infection after treatment, with no statistical heterogeneity in them $\left(\mathrm{P}=0.42, \mathrm{I}^{2}=0 \%\right)$. The results were analyzed by fixed effect model, and the pulmonary infection rate in the control group was obviously higher than that in the ANP treatment 
A

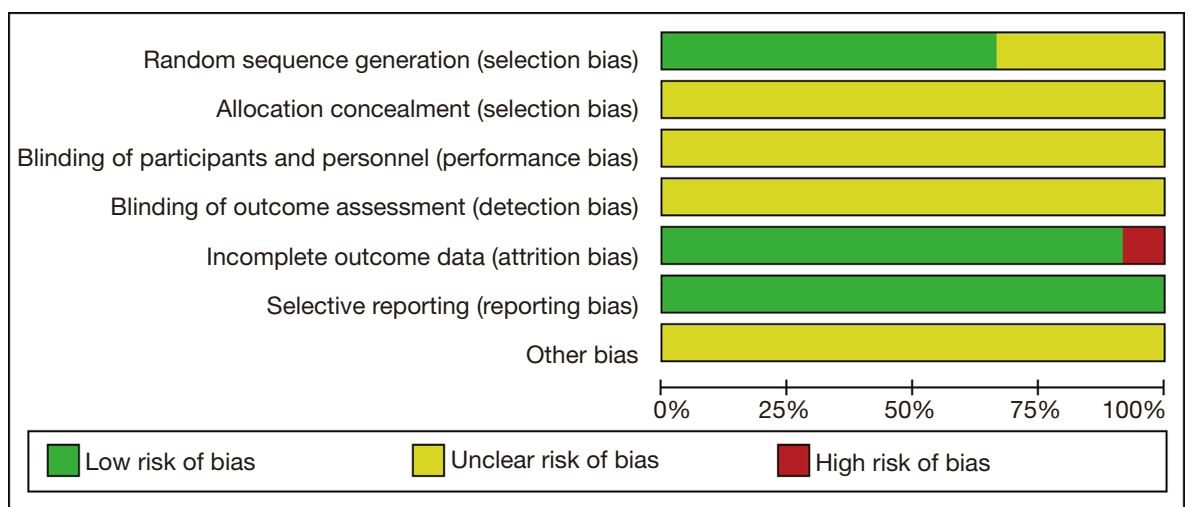

B

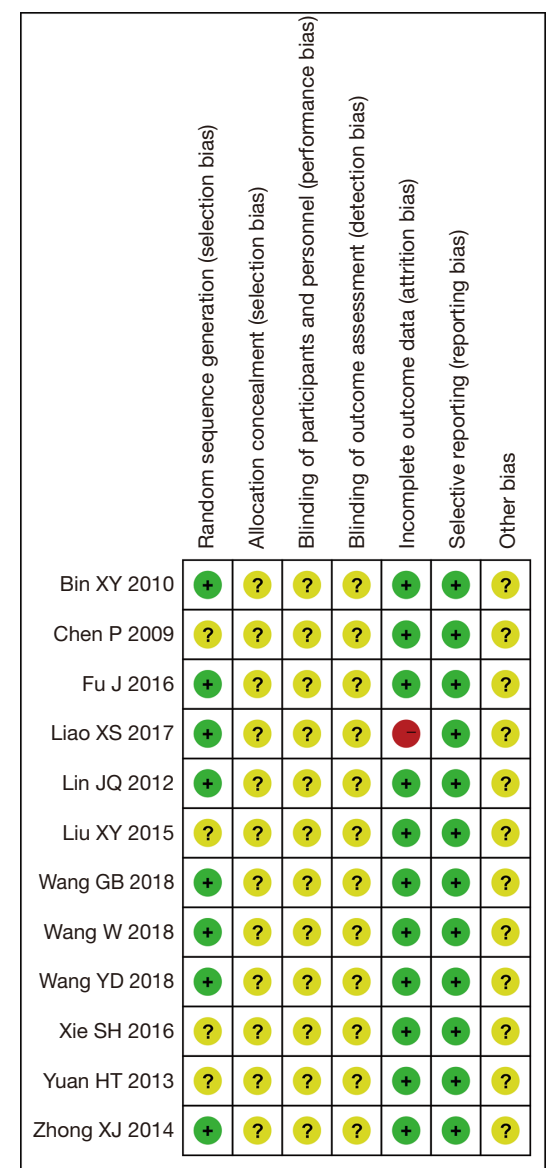

Figure 2 Graph of bias risk (A) and summary of bias risk (B). Review the author's judgment on each risk of bias for each included study.

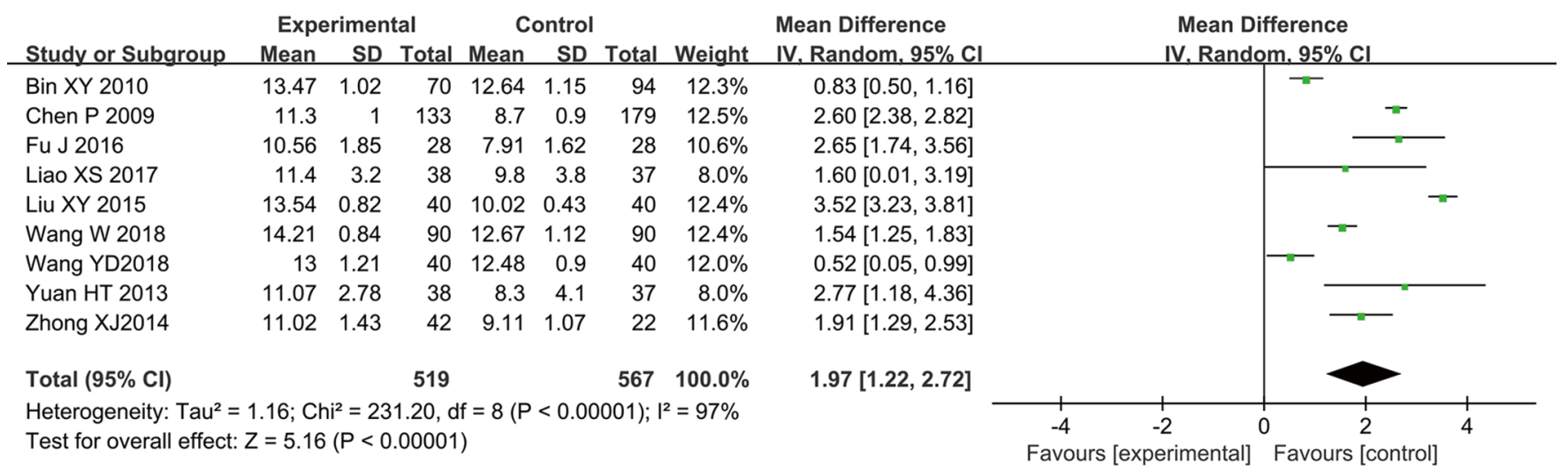

Figure 3 Forest plots of the GCS scores of the comparison between ANP and control groups. GCS, Glasgow Coma Score; ANP, Angong Niuhuang pill. 


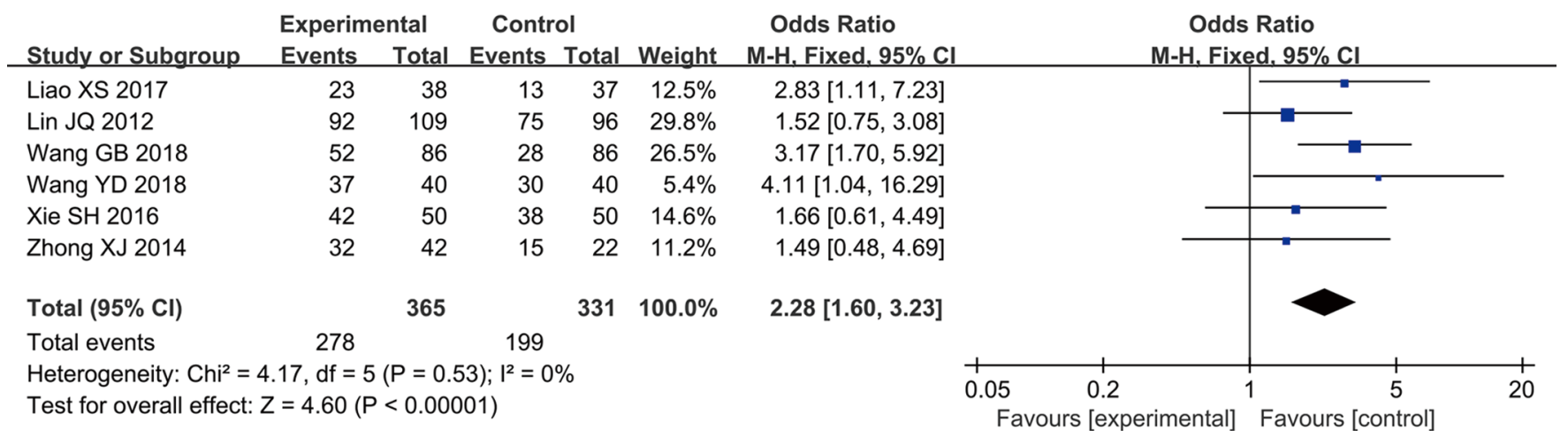

Figure 4 Forest plots of the GOS scores of the comparison between ANP and control groups. GOS, Glasgow Outcome Score; ANP, Angong Niuhuang pill.

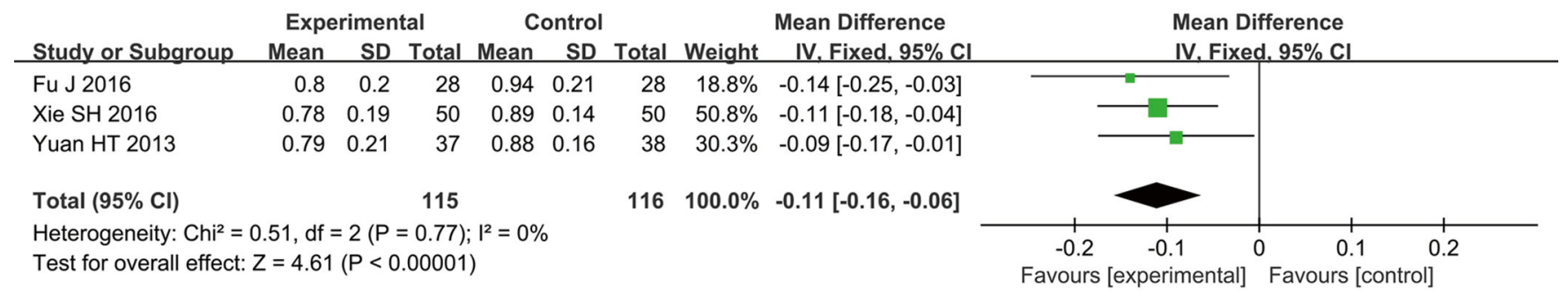

Figure 5 Forest plots of the concentration of $\mathrm{Mg}^{2+}$ of the comparison between ANP and control groups. ANP, Angong Niuhuang pill.

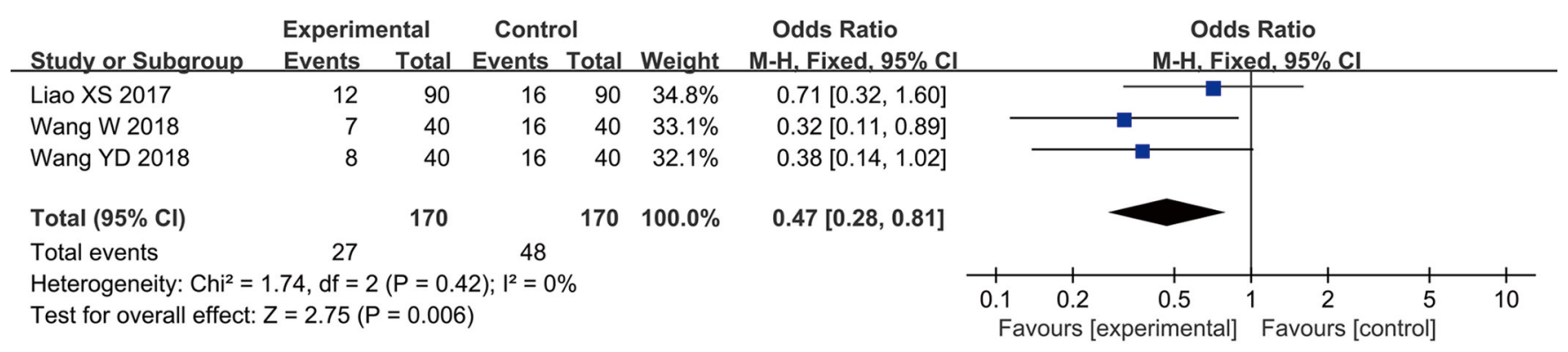

Figure 6 Forest plots of the incidence of pulmonary infection of the comparison between ANP and control groups. ANP, Angong Niuhuang pill.

group $[\mathrm{OR}=0.47,95 \% \mathrm{CI}(0.28,0.81), \mathrm{P}<0.01]$ (Figure 6).

\section{Qualitative description}

Two studies $(17,21)$ reported changes in NSE concentration after treatment, and both studies showed a significant difference in the ANP and control groups. Two studies $(18,21)$ reported gastrointestinal bleeding after treatment, and both studies showed that the ANP group had statistical significance in reducing gastrointestinal bleeding. Two studies $(18,21)$ reported hypohepatia after treatment, and both studies showed that the ANP group had statistical significance in reducing hypohepatia. Two studies $(21,22)$ reported renal insufficiency after treatment, and both studies showed that the ANP group had statistical significance in reducing renal insufficiency. 
Table 2 Publication bias evaluation of meta-analysis of ANP adjuvant therapy in the treatment of TBI

\begin{tabular}{|c|c|c|c|c|}
\hline Indicators & \multicolumn{2}{|c|}{ Begg's test } & \multicolumn{2}{|c|}{ Egger's test } \\
\hline GCS scores & 0.10 & 0.917 & -0.55 & 0.598 \\
\hline GOS scores & 0.00 & 1.000 & -0.10 & 0.928 \\
\hline The concentration of $\mathrm{Mg}^{2+}$ & 0.00 & 1.000 & 0.693 & 0.693 \\
\hline
\end{tabular}

ANP, Angong Niuhuang pill; TBI, traumatic brain injury; GCS, Glasgow Coma Score; GOS, Glasgow Outcome Score.

\section{Safety}

For all eligible trials, no adverse complications related to the administration of ANP were reported.

\section{Evaluation of sensitivity and publication bias}

A sensitivity analysis was performed after eliminating the articles one by one, and it was found that the results were stable, and there was no significant change. The Egger's regression and Begg's rank correlation tests suggested that there was no publication bias in all indicators $(\mathrm{P}>0.05)$ (Table 2).

\section{Discussion}

ANP is a commonly used prescription in traditional Chinese medicine. Modern pharmacological studies have shown that ANP can induce anti-inflammation, improve cerebral circulation, improve the tolerance of cerebral vessels to ischemia and hypoxia, protect brain cells, and reduce injury-induced brain edema (26). Previous studies have identified that ANP is widely used in patients with TBI and cerebral apoplexy (11). This study systematically evaluated the clinical efficacy of ANP in the treatment of the craniocerebral injury. The meta-analysis results showed that the GCS and GOS of ANP were significantly higher than those in the control group after the adjuvant treatment of the craniocerebral injury. This suggested that ANP can reduce a secondary brain injury and improve the prognosis of patients with the craniocerebral injury.

In this study, $\mathrm{Mg}^{2+}$ and NSE concentrations were analyzed. Some studies $(27,28)$ had shown that, through the severe craniocerebral trauma model, $\mathrm{Mg}^{2+}$ can increase the survival probability of neurons to protect neurons through a variety of protective mechanisms. These included reducing the formation of free radicals, blocking voltage-gated calcium channels and $\mathrm{N}$-methyl-D-aspartate channels, and inhibiting presynaptic excitatory nerves, which were closely linked to the prognosis of secondary brain damage in patients with severe brain injury. NSE is a specific enolase isoenzyme containing $\gamma$ subunits in neurons and neuroendocrine cells. NSE is released into the patients' blood with a severe brain injury after the bloodbrain barrier is damaged. The peripheral blood test results showed that NSE was abnormally increased (29). This study's results showed that the concentration of $\mathrm{Mg}^{2+}$ in the control group was lower than that in the experimental group, with significant difference. The other two studies on NSE reported significant differences between the ANP and control groups.

Despite the above benefits of ANP, the potential adverse effects of ANP should also be noted. None of the selected studies reported any adverse events in this systematic search and meta-analysis. In addition, this study analyzed the complications after ANP adjuvant therapy. The results suggested that ANP adjuvant therapy can significantly reduce pulmonary infection after craniocerebral injury, with a statistically significant difference. To compare gastrointestinal bleeding, liver function, and renal function, two studies reported that the ANP group can significantly reduce these complications. However, previous studies $(9,26)$ had shown that the hepatorenal toxicity potential of ANP should be monitored, especially for those who had committed themselves to long-time use. ANP should be used cautiously in patients with previous hepatorenal dysfunction. Therefore, the safety of ANP requires further research.

The limitations of this study were as follows. First, the number of the selected studies was limited, and all the selected trials were published in Chinese, which could have contributed to selection and publication bias. Second, the quality of the searched studies was low. Third, there 
were some differences in the dose and course of ANP pills. Finally, ANP itself has a strong, pungent smell and unique color, rendering the hiding of its distribution and double blindness in clinical trials difficult. Hence, the selection deviation cannot be ruled out. Due to the study's limitations, the above conclusions may lack reliability, which needs to be further verified by more scientific designs and rigorous implementation of large samples of RCT.

\section{Conclusions}

Based on limited evidence, ANP adjuvant therapy may have a clinical benefit in improving the prognosis of patients with TBI and reducing the associated complications. At the same time, more studies with larger sample sizes and high quality are required to determine the safety and effectiveness of ANP adjuvant therapy.

\section{Acknowledgments}

Thanks for the support from Hangzhou Huqingyutang Pharmaceutical Co., Ltd (batch Z33020160).

Funding: Key Research and Development Plan of human health in Anhui Province (No. 201904a07020108).

\section{Footnote}

Reporting Checklist: The authors have completed the PRISMA reporting checklist. Available at http://dx.doi. org/10.21037/apm-20-1331

Conflicts of Interest: All authors have completed the ICMJE uniform disclosure form (available at http://dx.doi. org/10.21037/apm-20-1331). The authors have no conflicts of interest to declare.

Ethical Statement: The authors are accountable for all aspects of the work in ensuring that questions related to the accuracy or integrity of any part of the work are appropriately investigated and resolved.

Open Access Statement: This is an Open Access article distributed in accordance with the Creative Commons Attribution-NonCommercial-NoDerivs 4.0 International License (CC BY-NC-ND 4.0), which permits the noncommercial replication and distribution of the article with the strict proviso that no changes or edits are made and the original work is properly cited (including links to both the formal publication through the relevant DOI and the license). See: https://creativecommons.org/licenses/by-nc-nd/4.0/.

\section{References}

1. Okonkwo DO, Shutter LA, Moore C, et al. Brain Oxygen Optimization in Severe Traumatic Brain Injury Phase-II: A Phase II Randomized Trial. Crit Care Med 2017;45:1907-14.

2. Johnson WD, Griswold DP. Traumatic brain injury: a global challenge. Lancet Neurol 2017;16:949-50.

3. Khellaf A, Khan DZ, Helmy A. Recent advances in traumatic brain injury. J Neurol 2019;266:2878-89.

4. Van de Zande N, Manivannan S, Sharouf F, et al. Demographics, presentation, and clinical outcomes after traumatic bifrontal contusions: a systematic review. Neurosurg Rev 2020;43:977-86.

5. Di BS, Wei M, Ma WJ, et al. A critical review to traumatic brain injury clinical practice guidelines. Medicine (Baltimore) 2019;98:e14592.

6. Lee B, Leem J, Kim H, et al. Herbal medicine for acute management and rehabilitation of traumatic brain injury: A protocol for a systematic review. Medicine (Baltimore) 2019;98:e14145.

7. Wang GB, Wang J. Study on the effect of Angong Niuhuang Pill in the treatment of severe craniocerebral injury. J Med Forum 2018;39:174-5.

8. Fan Q, Liu Y, Rao J, et al. Anti-Atherosclerosis Effect of Angong Niuhuang Pill via Regulating Th17/Treg Immune Balance and Inhibiting Chronic Inflammatory on ApoE/- Mice Model of Early and Mid-Term Atherosclerosis. Front Pharmacol 2020;10:1584.

9. Hou M, Wang X, Chen JH. Efficacy of Angong Niuhuang Pill in the Adjuvant Treatment of Ischemic Stroke in Acute Phase: A Meta-analysis. Chin Pharmacy 2016;27:5104-7.

10. Chai Y, Yin Z, Fan Q, et al. Protective Effects of Angong Niuhuang Pill on Early Atherosclerosis in ApoE-/- Mice by Reducing the Inflammatory Response. Evid Based Complement Alternat Med 2019;2019:9747212.

11. Guo Y, Yan S, Xu L, et al. Use of angong niuhuang in treating central nervous system diseases and related research. Evid Based Complement Alternat Med 2014;2014:346918.

12. Song H, Chen X, Yu Y, et al. Xingnao Kaiqiao acupuncture combined with Angong Niuhuang Wan for a patient under persistent vegetative state: a case report. Front Med 2018;12:334-9.

13. Liberati A, Altman DG, Tetzlaff J, et al. The PRISMA 
statement for reporting systematic reviews and metaanalyses of studies that evaluate health care interventions: explanation and elaboration. PLoS Med 2009;6:e1000100.

14. Higgins JP, Altman DG, Gøtzsche PC, et al. The Cochrane Collaboration's tool for assessing risk of bias in randomised trials. Version 2. BMJ 2011;343:d5928.

15. Bin XY. Clinical Observation on Treatment of Disturbance of Consciousness of Severe Herd Injury Patients by Angongniuhuang Pill. Shenzhen J Integrated traditional Chin and West Med 2010;20:367-9.

16. Chen P, Wang X. Observation on the Therapeutic effect of Angong Niuhuang Pill in the treatment of 133 cases of coma caused by brain Trauma. Zhejiang J Traditional Chin Med 2009;44:806.

17. Fu J. Clinical observation on the effect of Angong Niuhuang Pill on cooling, stopping smoking and promoting awakening in patients with severe brain injury. Pharmacology and Clinic of Traditional Chin Med 2016;32:162-4.

18. Liao XS, Luo Y, Yu L, et al. Clinical observation on Angong Niuhuang pill for adjuvant treatment of severe craniocerebral injury. Guangxi Med 2017;39:612-4.

19. Lin JQ. Angong Niuhuang Pill in the treatment of 109 cases of severe craniocerebral injury. Chin Pharmaceutical Industry 2012;21:107-8. doi: 10.3969/ j.issn.1006-4931.2012.22.068

20. Liu XY, Wang G. Effect of Angong Niuhuang Pill on Glasgow score in patients with acute severe craniocerebral trauma. Chin J Practical Neuro Dis 2015;18:82-4.

21. Wang W, Chen HF. Neuroprotective effect of Angong Niuhuang pill in severe traumatic brain injury patients and its effect on the permeability of enteric mucosa. J Bengbu

Cite this article as: Zhao PC, Huang ZS, Xu SN, Zhang YM. Adjuvant treatment with Angong Niuhuang pills in treating traumatic brain damage: a meta-analysis of randomized controlled trials. Ann Palliat Med 2021;10(2):1569-1577. doi: 10.21037/apm-20-1331
Med College 2018;43:201-5.

22. Wang YD, Zhang MW. Effect Observation of Standard Large Trauma Craniectomy Combined with Angong Niuhuang Pill for Treatment of Patients with Severe Traumatic Craniocerebral Injury. The latest Med Information Abstracts in the World 2018;18:38-39, 42.

23. Xie SH, Guo CW, Huang ZM, et al. Effect observation of modified Angong Niuhuang Pill in the treatment of severe craniocerebral injury. Chin Contemporary Med 2016;23:152-4.

24. Yuan WT, Huang LW, Xiang XW, et al. Effect of Angongniuhuang Pill on the Blood Magnesium Ion Concentration in the Acute Stage of Gravis Craniocerebral Injury. J Emerg in Tradi Chin Med 2013;22:1650-1.

25. Zhong XJ. Observation on the Application of Angong Niuhuang Pill in the treatment of moderate and severe craniocerebral injury. Med Theory and Practice 2014;27:185-6.

26. Liu H, Yan Y, Pang P, et al. Angong Niuhuang Pill as adjuvant therapy for treating acute cerebral infarction and intracerebral hemorrhage: A meta-analysis of randomized controlled trials. J Ethnopharmacol 2019;237:307-313.

27. Letson HL, Dobson GP. Adenosine, lidocaine, and $\mathrm{Mg} 2+$ $(\mathrm{ALM})$ resuscitation fluid protects against experimental traumatic brain injury. J Trauma Acute Care Surg 2018;84:908-16.

28. Lee SY, Kim SS, Kim CH, et al. Prediction of outcome after traumatic brain injury using clinical and neuroimaging variables. J Clin Neurol 2012;8:224-9.

29. Bagnato S, Andriolo M, Boccagni C, et al. Reduced Neuron-Specific Enolase Levels in Chronic Severe Traumatic Brain Injury. J Neurotrauma 2020;37:423-7. 